\title{
A case of erythema multiforme followed by herpes zoster
}

\author{
I. H. Park $\cdot$ J. N. Kang $\cdot$ J. E. Seol . \\ H. S. Sung $\cdot$ H. Kim
}

Received: 31 January 2014/Accepted: 11 April 2014/Published online: 24 April 2014

(C) Springer-Verlag Berlin Heidelberg 2014

A 59-year-old man presented with pruritic erythematous to violaceous patches on the whole body that had first appeared 1 week previously. Mucosa was intact and he had no other medical problem including herpes simplex virus (HSV) infection and had not taken any medication for several months. Physical examination revealed typical target lesions, which were symmetrically distributed over the entire body (Fig. 1). Histopathologic findings demonstrated interface dermatitis with dyskeratosis (Fig. 2). With the typical clinical and histopathologic features, erythema multiforme (EM) minor was diagnosed. Painful erythematous grouped vesicles appeared 4 days later on the right T9 and T10 dermatomes (Fig. 3). The serologic test showed positive for varicella-zoster virus (VZV) IgM and $\mathrm{IgG}$ which confirm the diagnosis of herpes zoster (HZ). We prescribed intravenous acyclovir in addition to systemic steroid for erythema multiforme, which improved the skin lesion.

Erythema multiforme is an acute mucocutaneous syndrome related to infection or medication with diverse mucocutaneous manifestation [1]. Most commonly identified predisposing factor is $\mathrm{HSV}$ [2]. HZ is the reactivation of latent VZV in sensory ganglia [3] which is rarely reported to be associated with erythema multiforme [4].
Twelve cases of VZV-associated EM have been reported in the literature. VZV infection preceded EM in ten cases and followed EM in the remaining two cases [1] as present case. The median interval between the onsets of the two distinct lesions was 9.3 days. HSV infection was excluded with negative serology for HSV and clinical features. Although incidental coincidence of EM and $\mathrm{HZ}$ could not be excluded, the VZV is thought to be a more plausible causative factor regarding temporal aspects.

Conflict of interest None.

\section{References}

1. Prais D, Grisuru-Soen G, Barzilai A, Amir J. Varicella zoster virus infection associated with erythema multiforme in children. Infection. 2001;29:37-9.

2. Weisman K, Petersen CS, Blichmann CW, Nielsen NH, Hultberg BM. Bullous erythema multiforme following herpes zoster and varicella-zoster virus infection. J Eur Acad Dermatol Venereol. 1998;11:147-50.

3. Chen SY, Suaya JA, Li Q, Galindo CM, Misurski D, Burstin S, Levin MJ. Incidence of herpes zoster in patients with altered immune function. Infection. 2014;42:325-34.

4. Wollina U, Gemmeke A. Herpes zoster-associated erythema multiforme. J Dermatol Case Rep. 2009;3:11-3.

I. H. Park · J. N. Kang · J. E. Seol · H. S. Sung · H. Kim $(\bowtie)$ Department of Dermatology, College of Medicine, Busan Paik Hospital, Inje University, Ga-geum 2 dong, Jin-gu,

Busan 633-165, Korea

e-mail: derma09@hanmail.net 


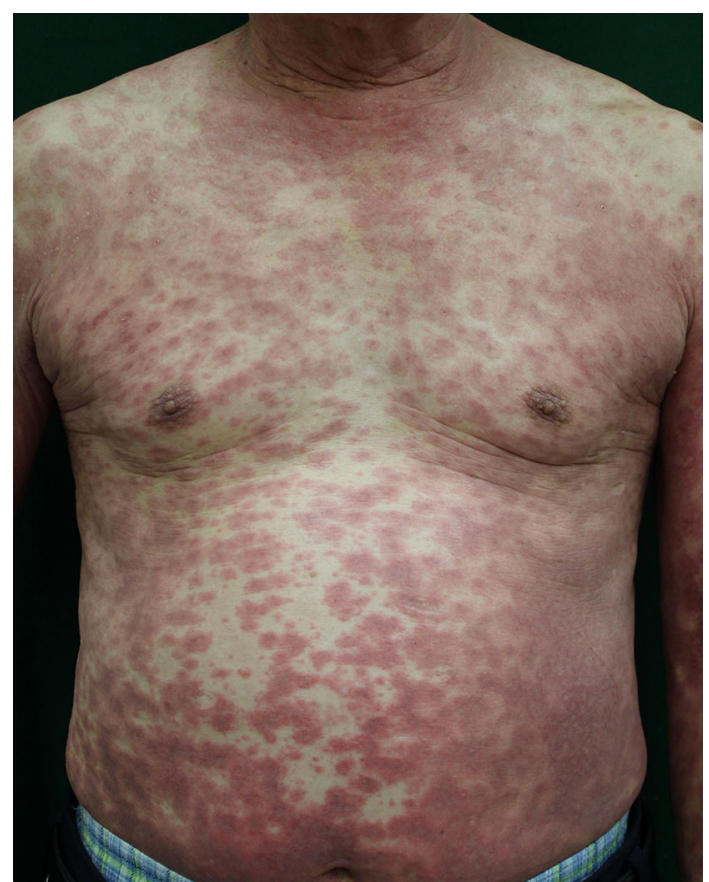

Fig. 1 Erythematous to violaceous targetoid annular patch on the trunk

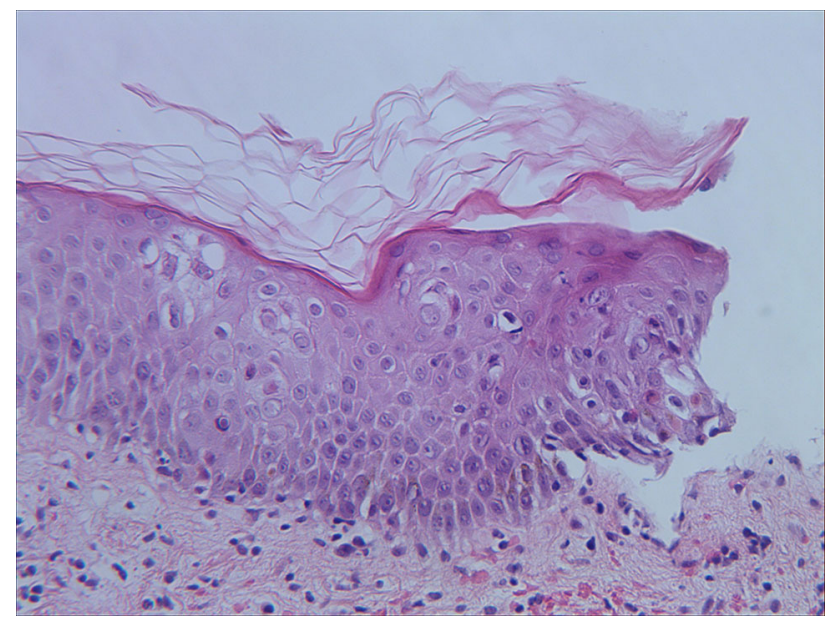

Fig. 2 Histopathologic findings revealed scattered necrotic keratinocytes, lymphocyte exocytosis, mild spongiosis in the epidermis, and inflammatory cell infiltration of the dermo-epidermal junction (H\&E, $\times 200)$

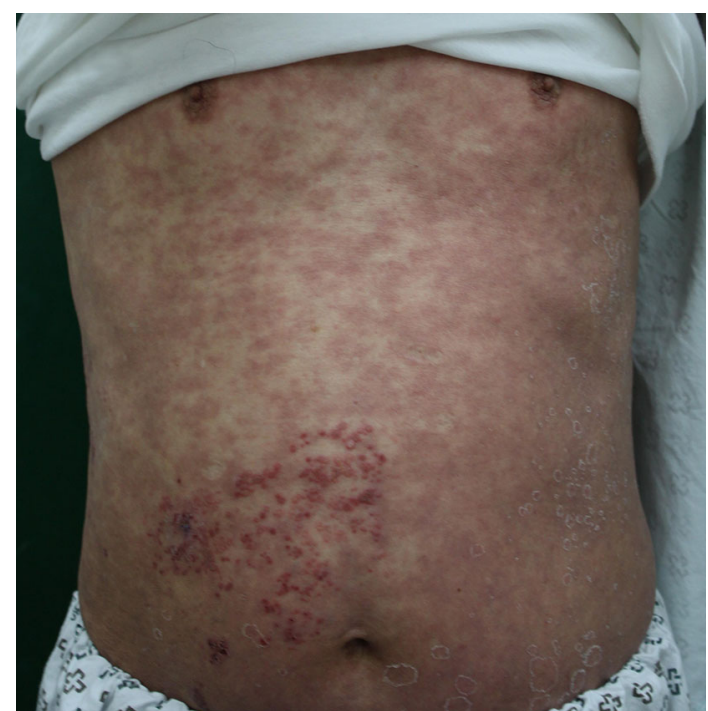

Fig. 3 Erythematous grouped vesicles along the right T9 and T10 dermatomes 